\title{
Desenvolvimento e Aplicação de uma Estratégia Pedagógica para o Ensino de Redes de Computadores com Robótica Educacional
}

\author{
Marcos Juares Vissoto Corino ${ }^{1}$, Silvia de Castro Bertagnolli ${ }^{1}$, Marcelo Augusto \\ Rauh Schmitt ${ }^{1}$ \\ ${ }^{1}$ Instituto Federal de Educação, Ciência e Tecnologia do Rio Grande do Sul (IFRS) \\ 90.030-041 - Porto Alegre - RS - Brasil \\ corino@veranopolis.ifrs.edu.br, \{silvia.bertagnolli, \\ marcelo.schmitt, \}@poa.ifrs.edu.br
}

\begin{abstract}
In this paper, we describe the development and application steps of a pedagogical strategy, which aims to provide the teaching of computer network concepts using Educational Robotics. For that, we chose the active project-based learning methodology and the theory of meaningful learning. We use the methodological procedures of bibliographic research, documentary research and case study. We identified as a result that the use of Educational Robotics, as a pedagogical alternative for the practical teaching of computer networks, maximized the performance of the participants.
\end{abstract}

Resumo. O presente artigo descreve o desenvolvimento e as etapas de aplicação de uma estratégia pedagógica que tem o intuito de propiciar o ensino de alguns conceitos de redes de computadores usando a Robótica Educacional. Para tanto, foi selecionada a metodologia ativa baseada em projetos, fundamentando-se na teoria da aprendizagem significativa. Os procedimentos metodológicos utilizados foram: pesquisa bibliográfica, pesquisa documental e estudo de caso. Como resultados identificados, tem-se que o uso da Robótica Educacional como alternativa pedagógica para o ensino prático de redes de computadores proporcionou uma melhora considerável no desempenho dos participantes da pesquisa.

\section{Introdução}

O crescimento da utilização de redes de computadores, fruto do processo de desenvolvimento das Tecnologias Digitais de Informação e de Comunicação (TDIC), ocorrido nas últimas décadas, demanda cada vez mais profissionais capacitados para manter e gerenciar serviços para que essas redes se mantenham em funcionamento, com bom desempenho, disponibilidade e segurança [Herpich et al. 2013]. As disciplinas de redes de computadores introduzem o aluno a um ambiente novo, onde são apresentados conteúdos que incluem muitos conceitos, protocolos, modelos de referência e uma vasta coleção de tecnologias [Cantú and Farines 2002].

O Ensino de Redes de Computadores (ERC) é prejudicado se os conceitos não são bem esclarecidos, tornando-se tedioso e cansativo quando a abordagem pedagógica se restringe à exposição dialogada [Herpich et al. 2013]. O ensino efetivo de redes de computadores requer experimentações com a finalidade de: situar o estudante sobre a pilha de protocolos; visualizar as características de hosts, enlaces e portas; e analisar o 
IX Congresso Brasileiro de Informática na Educação (CBIE 2020)

Anais do XXXI Simpósio Brasileiro de Informática na Educação (SBIE 2020)

comportamento dos periféricos em diferentes topologias e cenários [Belzarena and Gonzalez-Barbone 2008].

De modo a tornar o processo de ensino e de aprendizagem de conceitos de redes de computadores mais prático, o presente trabalho adotou a metodologia ativa de Aprendizagem Baseada em Projetos (ABP), pois com ela é possível envolver os alunos na construção de conhecimentos e competências utilizando projetos reais [Bacich and Moran 2018; Bender 2014]. Além disso, no presente trabalho optou-se por aplicar a teoria da aprendizagem significativa de Ausubel (2000), a qual expõe que a aquisição de novos significados requer um instrumento de aprendizagem significativa.

Para a parte da pesquisa descrita por este artigo, foram usados os seguintes procedimentos metodológicos: pesquisa bibliográfica, pesquisa documental e estudo de caso. A primeira concentrou-se em estudos sobre teóricos relacionados aos temas deste trabalho. A segunda foi realizada usando documentos do Instituto Federal de Educação, Ciência e Tecnologia do Rio Grande do Sul (IFRS), de modo a identificar os conteúdos de redes trabalhados nos Projetos Pedagógicos dos Cursos Técnicos em Informática do eixo de Informação e Comunicação do Catálogo Nacional de Cursos Técnicos (CNCT), operacionalizados no âmbito do IFRS. O estudo de caso contou com a participação de 14 estudantes do curso Técnico em Informática Subsequente ao Ensino Médio, do IFRS Campus Veranópolis. Para participar da pesquisa os participantes foram divididos em duas equipes, compostas por sete estudantes cada uma.

\section{Ensino de Redes de Computadores: estratégias possíveis}

O ensino de redes de computadores é desafiador, pois compreende uma série conceitos complexos que se distinguem da pedagogia tradicional, a qual emprega abordagens em sua maioria teóricas, não exprimindo a realidade prática do ensino de redes de computadores [Voss et. al. 2013]. De acordo com Herpich et al. [2013], "o ensino e aprendizagem de redes de computadores não é uma tarefa fácil"; e, quando os docentes priorizam o ensino de forma prática, os estudantes percebem maior relevância no processo de aprendizagem [Voss et. al. 2013].

Após investigação em repositórios de eventos, portais de teses e dissertações e bases de periódicos, é possível afirmar que existem diversas abordagens pedagógicas que podem ser aplicadas nesse contexto. $\mathrm{O}$ uso de laboratórios virtuais é apontado por Belzarena and Gonzalez-Barbone (2008), Ferreira et al. (2013) e Voss et al. (2013) como uma estratégia para conduzir as atividades práticas da disciplina de redes de computadores, pois tem como diferencial a possibilidade de o aluno realizar as atividades práticas em espaços que não estão restritos à sala de aula. O trabalho de Santos (2017) traz o uso de um ambiente em nuvem para o ERC através do simulador Netkit, possibilitando a realização das práticas de rede em ambiente de nuvem de computadores. Os trabalhos de Herpich (2013), Krassmann (2016) e Voss, Nunes e Medina (2013) propõem o uso de jogos sérios para complementar o processo de ensino e aprendizagem dos estudantes, disponibilizando atividades como simulações e resolução de problemas. O uso de agentes pedagógicos é empregado por: Freitas (2017), que usa agentes pedagógicos em um mundo virtual; e Herpich (2015), que aplica agentes inteligentes em ambientes virtuais imersivos tridimensionais (3D), visando a ampliar as alternativas para a construção do conhecimento dos alunos. 
Para Barbosa e Moura (2013, p. 62) "existe uma tendência atual de enaltecer a dimensão virtual, fortemente associada a informação, que pode provocar dificuldades e problemas complexos relacionados às necessidades de formação do indivíduo". Neste sentido a utilização de ambientes virtuais, realidade aumentada e simuladores pode aumentar a abstração do ensino, uma vez que são baseados em padrões matemáticos de causa-e-efeito (SETZER, 2002). Estes, em muitos casos não oportunizam a vivência de situações reais, as quais farão parte do cotidiano do profissional técnico em informática.

Deste modo, a adoção da robótica educacional foi utilizada como uma nova forma de trabalhar os conteúdos de redes de maneira prática, com a "mão na massa", ao invés do uso exclusivo de recursos virtuais, possibilitando explorar situações reais e contextuais do dia a dia do profissional técnico em informática. Com a adoção da RE, podem ser desenvolvidas atividades práticas que abordem os conceitos de diversas camadas dos modelos OSI e TCP/IP. No caso deste trabalho, a RE é usada para explicar comunicação com e sem fio, endereços físicos e lógicos, encaminhamentos, sockets e utilização de protocolos como HTTP (Hypertext Transfer Protocol), DNS (Domain Name System) e DHCP (Dynamic Host Configuration Protocol), demonstrando, de forma prática, a comunicação entre diferentes tipos de equipamentos, um robô e um PC, e de que forma os modelos se encaixam nessa tarefa.

\section{Aprendizagem Significativa: conceitos norteadores}

No desenvolvimento desta pesquisa, a teoria de Ausubel (2000), foi aplicada partindo-se das duas condições que devem ser consideradas para desencadear a aprendizagem significativa: (i) o estudante deve estar disposto a aprender, neste caso, os estudantes foram convidados a participar e foi explicitada a importância da disciplina e de que a experiência prática poderia favorecer o desenvolvimento de novos aprendizados e fortalecer a formação profissional; (ii) o conteúdo a ser aprendido tem que ser significativo: como o conteúdo da disciplina possui relação direta com a prática dos estudantes.

$\mathrm{Na}$ abordagem de Ausubel (2000), o professor possui um papel importante no processo da aprendizagem significativa, pois deve conduzir a aprendizagem considerando o conhecimento prévio do aprendiz, sendo necessário saber o que o aluno apreendeu sobre redes de computadores no decorrer de sua formação. Para isso, foram desenvolvidos testes iniciais que ajudaram a determinar quais conceitos deveriam ser novamente abordados, e quais já haviam sido assimilados. Isso possibilitou realizar o planejamento das aulas subsequentes, a que o pré-teste foi aplicado, concentrando-se nas relações que o aluno seria capaz de estabelecer.

Pode-se dizer que os conceitos de robótica educacional, em um primeiro momento, foram apresentados de maneira mecânica, pois o aluno dificilmente conseguia fazer a relação com os conteúdos aprendidos nas disciplinas, pois este é um assunto novo e não trabalhado anteriormente. A aprendizagem inicialmente mecânica, foi se tornando-se significativa à medida que foram utilizados organizadores prévios na forma de materiais introdutórios. Esta estratégia possibilitou que o novo conhecimento fosse mais facilmente relacionado com os subsunçores existentes na estrutura cognitiva do aluno, criando associação dos conceitos da robótica educacional com os sistemas de comunicação objetos desta pesquisa. 
Para favorecer a aprendizagem e estimular a diferenciação progressiva, o ensino foi organizado de forma que conceitos-chave da disciplina, como os modelos de camadas, fossem apresentados nas primeiras aulas e gradativamente particularizados, apontando suas diferenças, semelhanças e distinções no decorrer das demais, para que assim a reconciliação integrativa pudesse beneficiar o processo. Por fim, para verificar o quanto o aluno conseguiu assimilar foram aplicadas formas de avaliação, que permitiram determinar o grau em que os objetivos de aprendizagem estavam sendo alcançados.

\section{Procedimentos Metodológicos}

Esta pesquisa iniciou com um levantamento bibliográfico acerca dos temas relacionados ao trabalho, usando livros e artigos científicos para análise das variadas posições sobre o tema. Através do estudo, foi possível verificar o que estava sendo investigado e identificar os problemas e dificuldades na aprendizagem dos conceitos atinentes a redes de computadores. Essa etapa também permitiu fundamentar o trabalho usando os conceitos essenciais para a sua condução.

Em seguida, foi conduzida uma pesquisa documental, utilizando-se como base os Projetos Pedagógicos dos Cursos Técnicos de Informática, no âmbito do IFRS. Foi realizada uma análise das matrizes curriculares e dos planos de ensino dos seus componentes curriculares, mapeando-se os conteúdos trabalhados e generalizando-os sob um conceito comum, pois foram identificados conteúdos iguais sob nomenclaturas diferentes. Isso permitiu determinar os pontos semelhantes e que poderiam ser trabalhados com Robótica Educacional, os quais foram estruturados através de um mapa conceitual (Figura 1). Para abordar os conteúdos ilustrados no mapa conceitual foi utilizado o módulo ESP8266-01, porque disponibiliza uma solução completa e independente de rede $W i-F i$ : opera nos padrões $802.11 \mathrm{~b} / \mathrm{g} / \mathrm{n}$ e possui a pilha de protocolos TCP/IP integrada [Schwartz 2016].

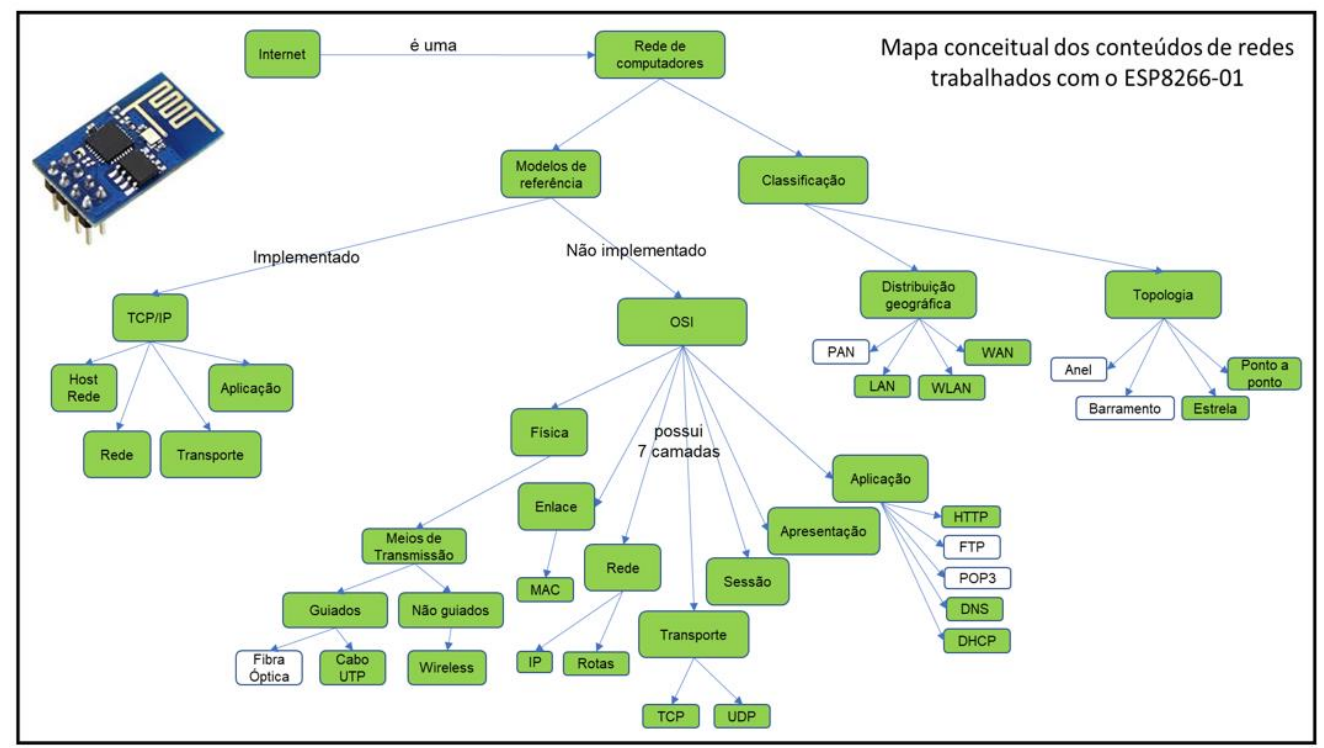

Figura 1. Mapa Conceitual: conteúdos trabalhados com o ESP8266-01.

Para validação da pesquisa, foi realizado um estudo de caso com 14 participantes, alunos matriculados na disciplina de Tópicos Especiais em Informática, do Curso Técnico em Informática Subsequente ao Ensino Médio, do IFRS Campus 
Veranópolis. A turma era composta majoritariamente de indivíduos do sexo masculino (73\%) trabalhadores, mas que em sua maioria atuava em áreas distintas a da Informática, porém com conhecimentos básicos de redes de computadores. Para o seu desenvolvimento foram utilizadas 07 aulas, durante sete semanas, totalizando 28 horas. Nesse período, foram abordados os temas de Redes de computadores que poderiam ser trabalhados com robótica educacional (Figura 1).

A execução da pesquisa ocorreu em quatro etapas distintas: pré-teste, planejamento, desenvolvimento das aulas, e teste final (Quadro 1). Os testes, compostos por vinte questões objetivas, abordaram temas de redes que fazem parte do cotidiano de um técnico em informática, tais como: aspectos das camadas física, de enlace e de rede; e, também, que poderiam ser trabalhados com robótica educacional. Foram abordados tópicos como meios de transmissão, arquiteturas e protocolos de redes, endereços físicos e lógicos, além de protocolos de aplicação. As questões aplicadas nos testes foram desenvolvidas com temas que seriam empregados no desenvolvimento do projeto. Os testes aplicados, no formato de questionário, foram usados como instrumentos de coleta de dados, contendo perguntas abertas e fechadas, as quais permitiram identificar os pontos positivos e negativos da prática desenvolvida. Cada aula foi planejada considerando a diferenciação progressiva e a reconciliação integrativa [Ausubel 2000], assim como alguns dos elementos previstos na ABP [Bender 2014].

\begin{tabular}{|c|c|c|c|}
\hline Pré-teste & Planejamento das aulas & Desenvolvimento das aulas & Teste final \\
\hline $\begin{array}{l}\text { Identificar o } \\
\text { que o aluno já } \\
\text { sabe sobre a } \\
\text { unidade de } \\
\text { aprendizagem. }\end{array}$ & $\begin{array}{l}\text { Planejar os conteúdos } \\
\text { abordados e os objetivos de } \\
\text { aprendizagem - Definir a } \\
\text { sequência dos conteúdos já } \\
\text { planejando a diferenciação } \\
\text { progressiva. } \\
\text { Estabelecer os materiais } \\
\text { didáticos introdutórios - } \\
\text { reconciliação integrativa entre } \\
\text { os conhecimentos prévios e os } \\
\text { novos. } \\
\text { Planejar o projeto que seria } \\
\text { desenvolvido, definição da } \\
\text { âncora e questão motriz, etc.. }\end{array}$ & $\begin{array}{l}\text { Utilizar diferenciação } \\
\text { progressiva, para facilitar a } \\
\text { ligação entre os conteúdos. } \\
\text { A avaliação com ênfase na } \\
\text { aprendizagem substantiva. A } \\
\text { avaliação considerou a } \\
\text { resolução do projeto } \\
\text { proposto; a relação que os } \\
\text { estudantes estabeleceram } \\
\text { entre os novos conceitos e } \\
\text { os pré-existentes; a } \\
\text { demonstração que } \\
\text { compreenderam a } \\
\text { diferenciação progressiva e a } \\
\text { reconciliação integrativa. }\end{array}$ & $\begin{array}{l}\text { Perguntas do teste final } \\
\text { devem estar } \\
\text { correlacionadas com } \\
\text { conteúdos abordados no } \\
\text { pré-teste. } \\
\text { Analisar se ocorreu ou } \\
\text { não evolução no } \\
\text { desempenho de cada } \\
\text { estudante. } \\
\text { Nesse momento os } \\
\text { estudantes preencheram } \\
\text { um questionário com } \\
\text { perguntas abertas e } \\
\text { fechadas sobre as suas } \\
\text { percepções da execução } \\
\text { da pesquisa. }\end{array}$ \\
\hline
\end{tabular}

Quadro 1. Etapas de Execução da Pesquisa.

A análise dos dados considerou os resultados obtidos com os testes aplicados, as questões que os integraram foram agrupadas sob categorias de acordo com o conteúdo abordado. Durante a análise, ocorreu a contabilização dos erros e acertos de cada aluno em cada uma das categorias, e os resultados obtidos foram cruzados usando-se tais categorias. Para organizar os resultados deste estudo, foram utilizadas a variação de acertos por conteúdo (divididos em geral e por aluno) e a variação de acertos por área e geral da turma. Na última aula foi realizada uma avaliação sobre toda a pesquisa e sobre o uso da robótica educacional.

\section{Relato da Experiência: aula a aula}

Para usar a ABP, é necessário estabelecer uma âncora que servirá para apoiar o ensino baseado em uma situação do mundo real [Bender 2014]. Para o planejamento desta pesquisa, o projeto partiu de uma demanda de docentes da área de física, vinculados à Educação Básica, do IFRS Campus Veranópolis. Então, foi definido como âncora um 
dispositivo robótico que viabilizasse o ensino de conceitos de física, especificamente do Movimento Circular Uniforme (MCU). Para tanto, foi selecionado, após várias discussões entre os pesquisadores, que seria utilizado um protótipo de um Chapéu Mexicano (brinquedo comum em parques de diversões), pois ele permite que os estudantes compreendam como atuam as forças centrípetas e centrífugas, que mantêm o movimento circular e elevam as cadeiras que fazem parte desse chapéu. Após várias reuniões, definiu-se que o protótipo do chapéu deveria ser capaz de: efetuar medições dos parâmetros de velocidade linear e angular, frequência, período e aceleração centrípeta; enviar as medições a um banco de dados; exibir as informações lidas e calculadas remotamente em uma página web; e gerar os gráficos de variação dos fenômenos capturados. A finalidade do protótipo é possibilitar que o docente de física possa fornecer aos alunos da Educação Básica exemplos visuais da ocorrência desse movimento, realizando experimentações sobre esses fenômenos, bem como demonstrando em tempo real os gráficos de variação desse movimento. Após definir o projeto as aulas foram conduzidas como descrito pelos próximos parágrafos.

$\mathrm{Na}$ aula 1, os alunos responderam ao pré-teste e com isso, foi possível verificar as lacunas na aprendizagem e os subsunçores que os sujeitos possuíam, para que os conceitos de Robótica Educacional pudessem ser correlacionados com os conceitos de redes de forma eficaz. Com os resultados desse teste, foi realizado o planejamento das aulas subsequentes com a finalidade de correlacionar os conceitos de redes com os de Robótica Educacional. Ainda na primeira aula, foi reapresentada a questão âncora e motriz, bem como os requisitos definidos para o projeto que seria desenvolvido. Após isso, os participantes reuniram-se em equipes, definiram os papéis de cada integrante e elencaram as atividades necessárias para o desenvolvimento do projeto, realizando todo o gerenciamento na ferramenta Trello.

Na aula 2, foram distribuídos os materiais para a montagem da infraestrutura de rede do projeto: computadores com a IDE Arduino e um computador servidor, roteador de banda larga, cabo UTP, conectores RJ45 e outras ferramentas. Foram trabalhados conceitos de classificação de redes, topologias, ativos de rede, meios de transmissão, camada física e servidores de rede, através de aula expositiva e dialogada. Para abordar os conceitos da área de redes, durante a montagem das estruturas, foram desenvolvidas atividades referentes aos meios de transmissão orientados, tais como os cabos UTP, seus tipos e os padrões de crimpagem. Nos meios de transmissão não guiados - a transmissão sem fio -, foram abordados conceitos de frequência, largura de banda, canais, padrões de segurança e processos de controle de acesso (MAC). Também foram feitas demonstrações sobre a topologia de rede utilizada e as classificações das redes com cabo (LAN), sem fio (WLAN), e sua interconexão com a internet (WAN). Nessa etapa, foram contemplados aspectos da camada física e de enlace do modelo OSI.

Na aula 3, foram distribuídos os módulos ESP8266 01, que permitem a ligação do Arduino a uma rede sem fio. Foram explicadas suas características e funções, abordando-se estudos de caso que demonstram o seu funcionamento. Os alunos fizeram a montagem do módulo de rede para realizar a comunicação do Arduino com sua infraestrutura. Em seguida, o professor explicou os conceitos de redes de computadores utilizados na comunicação do Arduino com o ESP e com a infraestrutura montada pelos alunos, situando-os sob a pilha de protocolos do Modelo OSI e TCP/IP, mais especificamente demonstrando os aspectos da camada de rede, como endereços IP e rotas, explicando como eles atuavam na comunicação entre esses dispositivos. Para isso, 
foram criados diversos exemplos, com o módulo ESP e o Arduino. Também se trabalhou com o protocolo da camada de aplicação DHCP, explicando-se como ele funciona e qual seria sua aplicação no projeto. Já na aula 4, os alunos trabalharam com a troca de dados entre o Arduino e a plataforma $W e b$ que era desenvolvida para a recepção de parâmetros do $\mathrm{MCU}$, realizando a geração de gráficos desse movimento. Nesse momento, foi possível trabalhar aspectos da camada de transporte, sessão e aplicação. Foi possível abordar utilização de Sockets, transmissão orientada e não orientada à conexão, estabelecimento de seções entre hosts e serviços HTTP (Hypertext Transfer Protocol) e métodos GET e POST, bem como troca de dados entre os dispositivos.

As três últimas aulas, foram organizadas desse modo: (i) aula 5, foi feita uma verificação acerca do andamento dos projetos de cada grupo, visando a identificar o nível de desenvolvimento e as dificuldades enfrentadas durante o processo. Diante dessas informações, foi conduzida uma nova explicação sobre os processos de comunicação, a infraestrutura montada e os demais pontos abordados nas aulas anteriores; (ii) aula 6, foi conduzido o desenvolvimento do projeto para que os alunos tivessem tempo hábil para atender a todos os requisitos. Durante a aula, o professor observou os grupos e indagou-os sobre os conceitos de redes utilizados. Se necessário, o docente explicava-os novamente para que o aluno entendesse o que estava acontecendo no processo de comunicação do Arduino com o servidor e vice-versa; (iii) aula 7, os alunos concluíram as tarefas, apresentaram para a turma a solução desenvolvida e realizaram uma atividade de reflexão/análise sobre todas as etapas da pesquisa. Logo após as apresentações, foi aplicado o teste final para avaliar se ouve ou não evolução nos conhecimentos dos estudantes com o desenvolvimento do projeto.

\section{Resultados e Discussões}

Com a adoção da RE, foi possível desenvolver atividades práticas que abordaram os conceitos de diversas camadas dos modelos OSI e TCP/IP. Este trabalho propôs o uso de comunicação com e sem fio, endereços físicos e lógicos, encaminhamentos, sockets e protocolos da camada de apresentação. Assim, foi possível demonstrar de forma prática a comunicação entre diferentes tipos de equipamentos, um robô e um PC, e de que forma os modelos e camadas da área de redes se encaixam nessa tarefa.

O desenvolvimento das atividades, com a utilização da robótica para o $\mathrm{ERC}$, foi bem aceito pelos estudantes, como comprovam os dados ilustrados no Figura 2. A maior parte dos alunos, 64\%, nunca tinha trabalhado com robótica antes. Após o desenvolvimento das atividades aqui descritas, todos os alunos afirmaram que a RE favoreceu a compreensão de vários aspectos teóricos da área de redes. Essa conclusão só foi possível, porque os estudantes já haviam cursado disciplinas de redes de computadores previamente, então, conseguiram estabelecer um comparativo entre o ERC com e sem o uso de robótica educacional. Os participantes indicaram que o uso de dispositivos não convencionais foi benéfico para entender as funções dos modelos de rede, mostrando de que forma os diversos tipos de equipamentos trocam informações tendo como base esses modelos. 


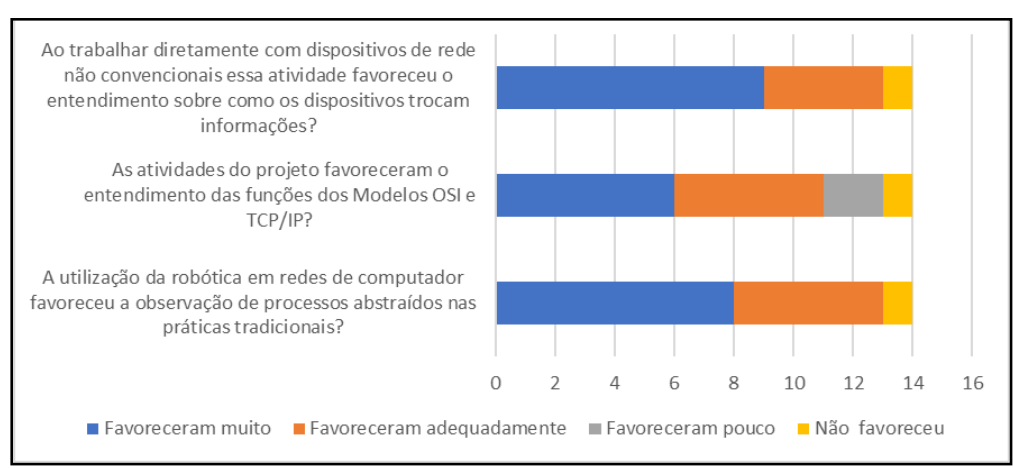

Figura 2. Percepções sobre a Robótica no ensino de redes

$\mathrm{O}$ ensino efetivo de redes de computadores requer experimentações com a finalidade de: situar o estudante sobre a pilha de protocolos; visualizar as características de hosts, enlaces e portas; e analisar o comportamento dos periféricos em diferentes topologias e cenários [Belzarena and Gonzalez-Barbone 2008]. Neste sentido, o uso da robótica educacional integrada a um projeto possibilitou que os conceitos apresentados pudessem ser mais bem compreendidos. De acordo com o que ilustra a Figura 3, tanto aspectos físicos quanto lógicos puderam ser entendidos de forma adequada pelos estudantes. Nota-se que, na Figura 2 o valor "1" corresponde à pouca compreensão, e o "5", à alta compreensão.

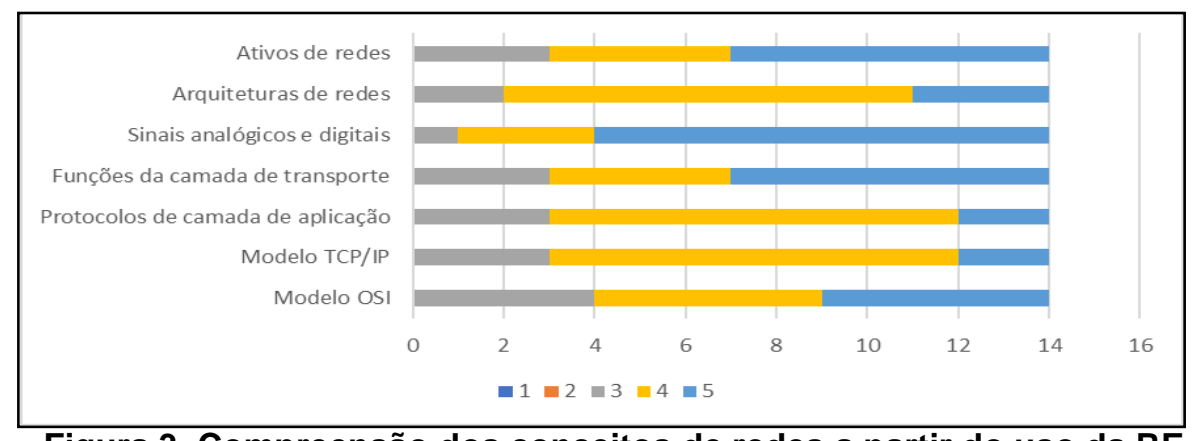

Figura 3. Compreensão dos conceitos de redes a partir do uso da RE

É possível verificar, no esquema da Figura 4, os acertos por conteúdo de forma geral. Fica evidenciado que o desempenho dos alunos nas questões que envolviam modelos foi melhor no teste final. Da mesma forma, as questões que envolvem protocolos da camada superior também foram favorecidas, pois sua utilização na Robótica Educacional se dá de forma diferenciada.

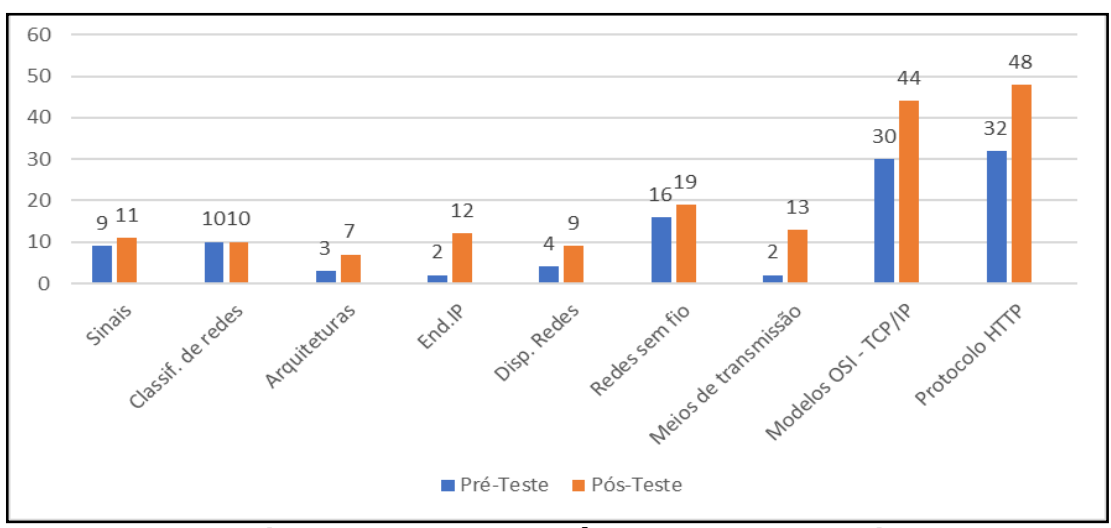

Figure 4. Acertos Pré-teste vs. Teste Final 
Observa-se, a partir da Figura 4, que, entre os assuntos abordados, aqueles que trabalham aspectos mais práticos que estão presentes no cotidiano do aluno, como classificação, dispositivos e arquiteturas de rede, não foram beneficiados pelo uso da RE da mesma forma que os assuntos que possuem uma abordagem mais teórica, como os modelos de redes.

Após a aplicação dos testes, percebeu-se um aumento de desempenho dos participantes. Enquanto no pré-teste a média de acertos geral foi de " 7,7 ", no teste final essa média foi de "12,4", considerando-se as 20 questões dos instrumentos avaliativos. Esse resultado é corroborado por Herpich et al. (2013): "o ensino e aprendizagem de redes de computadores não é uma tarefa fácil, [...] a prática é um fator de grande relevância no processo educacional".

\section{Conclusões}

$\mathrm{O}$ uso da teoria de aprendizagem significativa favoreceu o planejamento das aulas, pois como a organização dos materiais didáticos e das aulas expositivas foram organizadas com base nos princípios da diferenciação progressiva e da reconciliação integrativa. Além disso, os outros conceitos vinculados a essa teoria possibilitaram aos pesquisadores sistematizar o uso da RE de forma mais vinculada ao processo de aprendizagem dos estudantes. Mesmo estando dispostos a aprender e compreendendo que deveriam ter um papel ativo no seu processo de aprendizagem, percebe-se que um aspecto a ser vencido é a dificuldade em romper a passividade que alguns alunos demonstram quando, ao encontrarem uma dificuldade, não buscam a solução - ficam estagnados aguardando a resolução por parte do professor. Como esse é modelo é mais comum no seu cotidiano escolar, o estudante costuma apenas replicar a solução dada pelo docente, ele não está acostumado com a aprendizagem por descoberta.

Com o projeto proposto, os estudantes tiveram que implementar as funções de comunicação entre o objeto e o servidor, utilizar a tecnologia sem fio (Wi-Fi), e propiciar o que os dados ficassem acessíveis independentemente da plataforma utilizada. Para a montagem da infraestrutura de rede, eles usaram uma rede do tipo WLAN. No âmbito de redes de computadores, foram trabalhados, de forma prática, conceitos do modelo TCP/IP, desde a estrutura física da rede, endereços físicos e lógicos como MAC (Media Access Control) e IP respectivamente, protocolo TCP e sockets, até as funções da camada de aplicação, como controle de sessão e os protocolos de nível superior (HTTP, DNS, DHCP). Quanto à integração com o conteúdo das outras disciplinas, eles utilizaram conhecimentos de banco de dados, programação Web e programação $\mathrm{C}$, demostrando assim a interdisciplinaridade do projeto.

Por fim, pode-se afirmar que, a aplicação da Robótica Educacional se mostrou como uma nova forma de trabalhar os conteúdos de redes de modo prático, abordando situações reais e contextualizadas a partir da prática profissional. $\mathrm{O}$ uso da robótica educacional em sala de aula fez com que os estudantes não apenas utilizassem um dispositivo tecnológico, mas ela propiciou o desenvolvimento de algumas das habilidades necessárias para atuar no sociedade contemporânea, tais como: a criatividade, o trabalho em equipe, a colaboração, a resolução de problemas e a aquisição do conhecimento de modo interdisciplinar. 
IX Congresso Brasileiro de Informática na Educação (CBIE 2020)

Anais do XXXI Simpósio Brasileiro de Informática na Educação (SBIE 2020)

\section{Referências}

Ausubel, D. P. (2000) “Aquisição e Retenção de Conhecimentos: Uma perspectiva cognitiva", Platano.

Bacich, L.; Moran, J. (2018) "Metodologias Ativas para uma Educação Inovadora: Uma Abordagem Teórico-Prática”, Penso.

Barbosa, E. F. and Moura, D. G. De. (2013) "Metodologias Ativas de Aprendizagem na Educação Profissional e Tecnológica", Boletim Técnico do Senac, [s. 1.], v. 39, n. 2, p. 48-67, 2013.

Belzarena, P. and Gonzalez-Barbone, V. (2008) "Incorporacion de un Simulador Gráfico de Redes en un Objeto de Aprendizaje Reutilizablee-Spacio", UNED.

Bender, N. W. (2014) "Aprendizagem Baseada em Projetos: Educação diferenciada para o século XXI", Penso.

Cantú, E.; Farines, J. M. (2002) "Ensino-Aprendizagem de Redes de Computadores: Em Busca de uma Abordagem Metodológica Adaptada à Era da Internet", In: Anais do XXII Congresso da Sociedade Brasileira de Computação.

Ferreira, K. H. A. et al. (2013) "Laboratório Virtual para o Ensino de Redes de Computadores no Moodle”, In: Simpósio Brasileiro de Informática na Educação.

Freitas, V. C. B. (2017) “Agente pedagógico animado para o ensino de redes de computadores no mundo virtual TCN5", Dissertação de Mestrado, Portal Capes.

Herpich, F.; Jardim, R. R., Silva, R. F., Nunes, F. B., Voss, G. B. and Medina, R, D. (2013) "Jogos Sérios na Educação: Uma Abordagem para Ensino-Aprendizagem de Redes de Computadores (Fase I)", In: Nuevas Ideas en Informática Educativa TISE, http://www.tise.cl/volumen9/TISE2013/617-620.pdf.

Herpich, F. (2015) "ELAI: Intelligent agent adaptive to the level of expertise of students", Dissertação de Mestrado, Portal Capes.

Krassmann, A. L. (2016) "Jogo sério ubíquo integrado a mundo virtual opensim para o ensino de redes de computadores (JASPION)", Dissertação de Mestrado, Portal Capes.

Santos, S. L.; Silva, M. A. G. T. and Macedo, S. da H. (2012) "Mapas conceituais e aprendizagem significativa no ensino de rede de computadores do curso superior de telecomunicações”, In: RENOTE, v. 9, n. 2.

Setzer, V. W. (2002) “Computadores na educação: por quê, quando e como”. In: Meios Eletrônicos e Educação: uma visão alternativa. 2. ed. São Paulo: Escrituras, 2002.

Schwartz, M. (2016) “Internet of Things with ESP8266”, Packt Publishing.

Voss, G. B.; Nunes, F. B.; Medina, R. D. (2013) "Proposta de um jogo sério para o ensino de redes de computadores no ambiente virtual 3D OpenSim", In: Simpósio Brasileiro de Games.

Voss, G. B. et al. (2013) "Proposta de utilização de laboratórios virtuais para o ensino de redes de computadores: articulando ferramentas, conteúdos e possibilidades", In: RENOTE, v. 10, n. 2. 